\title{
ABORDAGEM E TRATAMENTO DO TABAGISMO NA ATENÇÃO PRIMÁRIA À SAÚDE
}

\author{
LOPES, R.P. ${ }^{1}$; CAVALCANTE, A.S.P. ${ }^{2}$; GOMES, D.F. ${ }^{3}$, RIBEIRO, M.A. ${ }^{4}$; MATHIAS, D.P. ${ }^{5}$ \& MACIEL, G.P. ${ }^{6}$
}

${ }^{1}$ Médico (UFCG). Especialista em Saúde da Família pela Universidade Federal do Ceará (UFC). E-mail: ronney_pinto@hotmail.com ${ }^{2}$ Enfermeira. Mestre em Saúde da Família pela Universidade Federal do Ceará (UFC). E-mail: anasuelen15@ hotmail.com ${ }^{3}$ Enfermeiro.

Mestre em Saúde da Família pela Universidade Federal do Ceará (UFC). E-mail: diogenesfgo@ gmail.com ${ }^{4}$ Enfermeiro. Mestre em Saúde da Família pela Universidade Federal do Ceará (UFC). E-mail: marcosaguiar61@ hotmail.com ${ }^{5}$ Médico pela Universidade Federal de

Campina Grande. E-mail: diegopmathias@gmail.com ${ }^{6}$ Graduando em Enfermagem na Universidade Estadual Vale do Acaraú. E-mail: gabrielpmaciel12@gmail.com

Artigo submetido em 24/07/2018

\begin{abstract}
RESUMO
Este estudo teve como objetivo descrever a experiência da implementação da abordagem e tratamento do tabagismo na Atenção Primária à Saúde (APS) de um município nordestino, por meio de grupo operativo. Trata-se de um relato de experiência sobre as ações de controle tabágico, seguindo as etapas de (i) realização de busca ativa de fumantes no território, (ii) acolhimento e primeiro atendimento, (iii) estratificação quanto ao estágio motivacional para mudança, (iv) realização das sessões de grupo para cessação tabágica e (v)
\end{abstract}

instituição da farmacoterapia. Durante os encontros, utilizou-se a abordagem ativa por meio da problematização, com ênfase nos principais conhecimentos que o fumante deve incorporar. A implementação da abordagem e tratamento do tabagismo no âmbito da APS permitiu, num primeiro passo, realizar o diagnóstico situacional de uma população específica e em território determinado, processo importante para o levantamento de problemas e construção de ações qualificadas e abrangentes.

PALAVRAS-CHAVE: Tabagismo; Abandono do Uso de Tabaco; Atenção Primária à Saúde.

\section{APPROACHING AND TREATING SMOKING AT PRIMARY HEALTH CARE}

\section{ABSTRACT}

This study aimed to describe the experience of the implementation of the approach and treatment of smoking in the Primary Health Care (PHC) of a northeastern municipality, through an operating group. This is an experience report about the actions of tobacco control, following the steps of (i) conducting an active search for smokers in the territory, (ii) reception and first care, (iii) stratification regarding the motivational stage for change, (iv) conducting group sessions for smoking cessation and (v) institution of pharmacotherapy. During the meetings, the active approach was used through the problematization, with emphasis on the main knowledge that the smoker should incorporate. The implementation of the approach and treatment of smoking in the context of PHC allowed, in a first step, to carry out the situational diagnosis of a specific population and in a determined territory, an important process for the survey of problems and construction of qualified and comprehensive actions.

KEYWORDS: Tobacco Use Disorder; Tobacco Use Cessation; Primary Health Care.

\section{INTRODUÇÃO}

O tabagismo, apontado como segundo principal fator de risco para mortalidade prematura e incapacidades, representa um grave problema de saúde, sobretudo por sua elevada prevalência em todo o mundo (BRASIL, 2015a; REITSMA, et al, 2017). Atualmente, afeta cerca de 1,1 bilhão de pessoas, número equivalente a $21 \%$ da população global com idade 
superior a 15 anos, sendo responsável, anualmente, por quase 6 milhões de mortes, das quais mais de 600 mil decorrem da exposição passiva ao fumo (ACHARYA, et al, 2016).

O tabagismo ativo e passivo expõe o indivíduo ao contato com mais de 4.700 substâncias agressivas ao organismo, sendo mais de 60 delas potencialmente cancerígenas (BRASIL, 2015b; SATTLER, 2011). Portanto, está estabelecida sua associação direta com doenças do aparelho circulatório (doença cardíaca isquêmica e cerebrovascular); câncer de traqueia, brônquios e pulmão, esôfago, estômago, fígado, pâncreas, rim e ureter, colo uterino, bexiga e colorretal; doenças respiratórias crônicas (asma e doença pulmonar obstrutiva crônica) e metabólicas (diabetes mellitus) (BRASIL, 2015b; REITSMA, et al, 2017; ACHARYA, et al, 2016; SATTLER, 2011)

No Brasil, ainda que de forma pouco articulada, o primeiro Programa Nacional contra o Fumo foi criado em julho de 1979, estimulado, sobretudo, por estudos pioneiros de caráter nacional que demonstravam o aumento de doenças tabaco-relacionadas (ROMERO; COSTA E SILVA, 2011).

Porém, a política brasileira de controle do tabagismo apresentou seu maior avanço em 1989, quando a governança das ações foi transferida para o Instituto Nacional do Câncer (INCA), que passou a conduzir o Programa Nacional de Controle do Tabagismo (PNCT). O programa, incluindo ações legislativas, econômicas e educativas, tem como objetivo reduzir a prevalência de fumantes no país e a morbimortalidade relacionada ao problema (BRASIL, 2015a; ROMERO; COSTA E SILVA, 2011).

As atuais diretrizes do PNCT incluem: (i) redução da iniciação do consumo de tabaco, (ii) proteção da população contra os riscos do tabagismo passivo, (iii) redução da aceitação social do tabagismo, (iv) aumento do acesso ao tratamento do fumante, (v) controle e monitoramento dos produtos derivados do tabaco e (vi) monitoramento das tendências de consumo/estratégias da indústria (CAMPOS; GOMIDE, 2015).

No escopo da legislação federal brasileira que integra o PNCT, o tratamento da pessoa tabagista, no âmbito da assistência à saúde, assume papel de destaque somente com a aprovação da Portaria n 1035, de 31 de maio de 2004, do Ministério da Saúde (MS), que ampliou o acesso à abordagem e ao tratamento do tabagismo para a rede da atenção primária e de média complexidade do Sistema Único de Saúde (SUS) (BRASIL, 2004a). Em 13 de agosto do mesmo ano, publicou-se ainda a Portaria $\mathrm{n}^{\circ} 442$, do MS, aprovando o Plano para Implantação da Abordagem e Tratamento do Tabagismo no SUS e o Protocolo Clínico e Diretrizes Terapêuticas - Dependência à Nicotina (BRASIL, 2004b). 
Destaca-se ainda a Portaria MS nº 571, de 5 de abril de 2013, que atualizou as diretrizes do tratamento da pessoa tabagista no âmbito do SUS, reforçando a Atenção Primária à Saúde (APS) enquanto lócus principal de cuidado e sensível ao desenvolvimento de ações de estímulo e apoio à adoção de hábitos de vida saudáveis (BRASIL, 2013).

Nesse contexto, os programas de valorização, formação, provimento e fixação de profissionais de saúde, instituídos na última década pelo Ministério da Saúde (MS) como um esforço para enfrentar os desafios que vinham condicionando o desenvolvimento da Atenção Primária no País, têm possibilitado também ampliar e fortalecer ações de informação, incentivo e apoio ao abandono do tabagismo. O Programa Mais Médicos para o Brasil (PMM) e o Programa de Valorização do Profissional da Atenção Básica (Provab) integram essa estratégia.

Identificou-se, portanto, no exercício da atuação médica vinculada ao Provab em um município de médio porte do estado do Ceará, a presença de elevado número de fumantes que identificam o tabagismo como problema, mas não têm encontrado apoio necessário dos profissionais de saúde sobre como parar de fumar, tampouco a possibilidade de acompanhá-los nesse processo.

Nesse sentido, este relato teve por objetivo descrever as experiências da implementação da abordagem e tratamento do tabagismo na APS de um município nordestino.

\section{A ATENÇÃo PRIMÁRIA À SAÚde COMO LóCUS PRIVILEGIAdO NO CONTROLE DO TABAGISMO}

Estudo de Reitsma et al. (2017) concluiu que, entre os dez países com o maior número de tabagistas com consumo diário, o Brasil apresentou a maior redução global da prevalência tanto para a população do gênero masculino $(56,5 \%)$ quanto feminino $(55,8 \%)$, comparando os anos de 1990 e 2015.

Parte expressiva dessa evolução pode ser atribuída ao Programa Nacional de Controle do Tabagismo, que foi instituído em 1989 com o objetivo de reduzir a prevalência de fumantes e a morbimortalidade associada ao consumo do tabaco (CARGNELUTTI; BRUM; ALÓS, 2014).

A Atenção Primária à Saúde (APS) configura-se como espaço privilegiado e estratégico para o controle do tabaco e seus derivados, na perspectiva do Sistema Único de Saúde, por se caracterizar como:

[...] um conjunto de ações de saúde, no âmbito individual e coletivo, que abrange a promoção e a proteção da saúde, a prevenção de agravos, o diagnóstico, o tratamento, a reabilitação, a redução de danos e a manutenção da saúde com o objetivo de 
desenvolver uma atenção integral que impacte na situação de saúde e autonomia das pessoas e nos determinantes e condicionantes de saúde das coletividades (BRASIL, 2011a).

Além disso, Giovanella (2008), em sua reflexão acerca das diferentes concepções de Atenção Primária nos sistemas nacionais de saúde, considera que a assistência integral às condições clínicas crônicas, a exemplo do tabagismo, é relevante nesse nível assistencial, uma vez que:

[...] a coordenação e a longitudinalidade, atributos de uma Atenção Primária à Saúde robusta, como proposto por Starfield, são qualidades assistenciais imprescindíveis para garantia da adesão e continuidade do cuidado [...] produzindo estratégias de cuidados menos invasivas e mais dialógicas com produção de autonomia para os sujeitos como suportado pelo "eixo democrático/participativo" da Atenção Primária à Saúde (GIOVANELLA, 2008).

Dessa forma, por meio de seus quatro atributos essenciais, que são (1) o Primeiro Acesso, (2) a Integralidade, (3) a Longitudinalidade, e (4) a Coordenação do Cuidado, pretendese que a APS seja resolutiva e garanta um cuidado de forma qualificada e abrangente (BRASIL, 2015a).

A partir do Primeiro Acesso, o usuário identifica nesse nível de atenção o lócus privilegiado a cada novo problema ou a cada nova demanda que interfira direta ou indiretamente no seu processo de saúde e adoecimento, além de pressupor maior vínculo entre profissional/serviço de saúde e usuário do Sistema Único de Saúde (STARFIELD, 2002).

O Ministério da Saúde, através da Portaria MS/GM nº. 571, de 5 de abril de 2013, define como atribuições gerais da Atenção Primária à Saúde para prevenção e tratamento do tabagismo (BRASIL, 2013):

1. Realizar ações de promoção da saúde e de prevenção do tabagismo de forma intersetorial e com participação popular;

2. Identificar as pessoas tabagistas que fazem parte da população sobre sua responsabilidade;

3. Realizar a avaliação clínica inicial;

4. Prestar assistência terapêutica e acompanhamento individual e/ou em grupo, abrangendo desde a abordagem mínima até a abordagem intensiva, acompanhadas se necessário de tratamento medicamentoso;

5. Organizar a realização de consultas e grupos terapêuticos para as pessoas tabagistas;

6. Disponibilizar os medicamentos para o tratamento do tabagismo de acordo com a Relação Nacional de Medicamentos Essenciais (RENAME);

7. Diagnosticar e tratar precocemente as possíveis complicações decorrentes do tabagismo; e

8. Acionar o Programa Nacional Telessaúde Brasil Redes ou outra estratégia local, sempre que necessário, para qualificar a atenção prestada (BRASIL, 2013).

\section{MATERIAIS E MÉTODOS}

Trata-se de um projeto de intervenção de uma abordagem com pacientes tabagistas na 
Atenção Primária à Saúde. O estudo teve como abordagem, a qualitativa, cujo objetivo é a descrição de determinada experiência com a pretensão de contribuir de forma relevante para um dado objeto de estudo ou campo do conhecimento (GIL, 2010). Esse encaminhamento investigativo condiz, portanto, com o propósito de caracterizar o perfil da população tabagista de determinado território e o tratamento para cessação adequado à proposta do Ministério da Saúde, a partir de uma abordagem integral no nível primário de atenção.

Elegeu-se uma unidade de saúde da zona urbana para a realização das experiências, a partir de critérios sociodemográficos e corresponsabilização da equipe de saúde no desenvolvimento da intervenção. A intervenção ocorreu no período de 2 meses para que pudessem ser realizadas as seguintes ações de desenvolvimento das ações: (i) reconhecimento da pessoa tabagista no território adstrito à unidade de saúde; (ii) acolhimento e primeiro atendimento do usuário; (iii) estratificação quanto ao estágio motivacional para a cessação tabágica; (iv) execução da estratégia de grupo para abandono do tabaco (abordagem cognitivocomportamental); e (iv) avaliação da necessidade e disponibilidade de terapia medicamentosa (abordagem farmacológica).

Para implementação das ações, foram utilizados como critérios de seleção dos atoresalvo: ter idade superior a 18 anos; fazer o consumo diário ou ocasional de produtos derivados de tabaco; encontrar-se no estágio motivacional de contemplação, preparação, ação, manutenção ou recaída, segundo o Modelo de Avaliação do Grau de Motivação para a Mudança (PROCHASKA; DICLEMENTE; NORCROSS, 1992); e ter disponibilidade de tempo para participar dos grupos de cessação do tabagismo. Foram excluídas as pessoas identificadas no estágio de pré-contemplação ou que já se encontravam em abstinência do tabaco no momento da abordagem inicial.

O público-alvo da intervenção constituiu-se por 40 usuários tabagistas foram identificados e concordaram em participar da intervenção, cujas ações foram inicialmente implementadas pela equipe de saúde da família (médico, enfermeiro, técnico de enfermagem e 10 agentes comunitários de saúde). Posteriormente, foram incorporados 3 profissionais (psicólogo, fisioterapeuta e nutricionista) do Núcleo de Apoio à Saúde da Família (NASF).

Sendo a avaliação individual do fumante uma condição necessária para a efetividade das ações de controle tabágico, utilizou-se um instrumento padronizado contendo os dados de identificação e sociodemográficos, história tabagística e patológica pregressa, o Questionário de Fagerström (FAGERSTRÖM, 1978), a Escala de Razões para Fumar modificada, e espaço para registro do exame físico do usuário. 
A aplicação da Escala de Razões para Fumar modificada identifica qualitativamente as situações que levam o sujeito ao consumo do tabaco, agrupando-as em nove fatores principais: dependência, prazer de fumar, redução da tensão, estimulação, automatismo, manuseio, tabagismo social, controle de peso e associação estreita (BRASIL, 2015b; SOUZA, et al, 2009; VIEIRA, 2014).

A avaliação do grau de motivação para a mudança seguiu o modelo proposto por Prochaska, Diclemente e Norcross (1992), relevante na adequação das intervenções terapêuticas propostas ao usuário e fundamentado em seis estágios: pré-contemplação, contemplação, preparação, ação, manutenção e recaída.

As ações direcionadas ao tratamento para cessação do tabagismo compreenderam a realização de intervenções psicossociais e farmacológicas. A abordagem cognitivocomportamental intensiva, através da modalidade de tratamento em grupo, constituiu-se como alicerce terapêutico.

Para instituição da terapia medicamentosa, utilizou-se como parâmetros o perfil tabagístico, presença de comorbidades e tentativa anterior de cessação. Para escolha, foram observados também os fármacos disponíveis na Relação Nacional de Medicamentos Essenciais de 2014 (Rename) (BRASIL, 2015b).

Esperou-se que com a intervenção, o número de usuários tabagistas pudesse ser diminuído e melhorado a qualidade de vida desses pacientes. Os resultados alcançados com as ações foram analisados com base na frequência absoluta, relativa e percentual. Os dados de identificação dos usuários participantes da intervenção foram mantidos em sigilo para atender às diretrizes sobre pesquisa envolvendo seres humanos preconizadas pelo Conselho Nacional de Saúde em sua Resolução CNS n 466, de 12 de dezembro de 2012.

\section{RESULTADOS E DISCUSSÕES}

Aderindo uma interpretação sobre o contexto de qualificação profissional e organização do planejamento em saúde, foi realizada educação permanente com a equipe multiprofissional, buscando depreender os conhecimentos prévios acerca do tabagismo e articulando-os com novos conceitos atrelados à temática. Após esse momento, realizou-se o reconhecimento da população tabagista no território adstrito, através da busca ativa em visita domiciliar e durante a realização de consultas de rotina com o médico e enfermeiro.

Quanto ao perfil sociodemográfico dos 40 fumantes avaliados, predominou: sexo feminino em 28 sujeitos (70\%); faixa etária entre 45 e 64 anos, com 22 sujeitos (55\%); $1^{\circ}$ grau 
incompleto e completo de escolaridade, com 28 sujeitos (70\%); e 18 (45\%) casados.

A aplicação do Questionário de Tolerância de Fagerström revelou que mais de 50\% dos participantes apresentaram nível de dependência à nicotina elevado e muito elevado. Além disso, 23 participantes $(57,5 \%)$ referem fazer uso, em média, de 11 a 20 cigarros por dia. No tocante ao tempo gasto para acender o primeiro cigarro, a maioria dos fumantes (70\%) inicia o consumo nos primeiros trinta minutos do dia.

A avaliação qualitativa dos usuários, por meio da Escala de Razões para Fumar modificada, demonstrou que mais de $50 \%$ deles apresentaram os seguintes fatores de atenção (escore final superior a dois): dependência, prazer de fumar, redução da tensão (relaxamento), estimulação, manuseio (ritual), tabagismo social e associação estreita. Esses fatores representam os principais domínios motivacionais associados ao tabagismo encontrados nos participantes. Redução da tensão e controle do peso apresentaram, respectivamente, maior (90\%) e menor (30\%) frequência com a aplicação da escala, conforme mostra a Tabela 2 a seguir.

Tabela 2. Caracterização do perfil de dependência à nicotina de 40 participantes, agrupada em nove fatores principais, segundo a Escala de Razões para Fumar modificada. Crateús, 2017

\begin{tabular}{lccc}
\hline Fatores de Atenção (escore $\geq 2)$ & Frequência $\left(f_{\mathrm{i}}\right)$ & $\begin{array}{c}\text { Frequência } \\
\text { relativa }\left(f_{\text {ri }}\right)\end{array}$ & $\begin{array}{c}\text { Frequência } \\
\text { percentual }\end{array}$ \\
\hline Redução da tensão (tensionreduction) & 36 & 0,9 & 90 \\
Dependência (addiction) & 34 & 0,85 & 85 \\
Prazer de fumar (pleasure) & 31 & 0,775 & 77,5 \\
Associação estreita (affiliativeattachment) & 30 & 0,75 & 75 \\
Estimulação (stimulation) & 30 & 0,75 & 75 \\
Manuseio (handling) & 22 & 0,55 & 55 \\
Tabagismo social (social smoking) & 22 & 0,55 & 55 \\
Automatismo (automatism) & 19 & 0,475 & 47,5 \\
Controle de peso (weightcontrol) & 12 & 0,3 & 30 \\
\hline
\end{tabular}

FONTE: Elaborado pelos autores.

No processo da abordagem intensiva da pessoa tabagista, adaptou-se a proposta do Consenso de Abordagem e Tratamento do Fumante (BRASIL, 2001). Seguiu-se a proposição de quatro sessões estruturadas de 90 minutos cada, ampliando-se posteriormente para oito, por decisão compartilhada e motivada pelos usuários.

As temáticas utilizadas nas quatro primeiras sessões foram, nessa ordem: (i) entender por que se fuma; (ii) preparar para abandono do cigarro; (iii) viver os primeiros dias sem o 
cigarro; e (iv) vencer obstáculos e manter-se sem fumar. Para a realização dos últimos quatro encontros de acompanhamento, buscou-se compartilhar conquistas e dificuldades, além de prevenir recaídas quando em abstinência.

Durante a estratégia de grupo, 4 (10\%) abandonaram o tratamento, sendo todos os casos ocorridos entre a segunda e terceira sessões. Em relação à abordagem farmacológica, 21 $(58,3 \%)$ participantes fizeram uso de algum tipo de medicamento disponível na Rename 2014 (BRASIL, 2015b).

Sobre a caracterização da abstinência ao tabaco, a partir da realização do tratamento integral proposto, $27(67,5 \%)$ estavam abstinentes até a última sessão, 5 (12,5\%) não abandonaram o hábito e 4 (10\%) apresentaram recaída, conforme Tabela 3 apresentada a baixo. Aos 9 fumantes que, ao final das oito sessões, ainda se encontravam sob uso do tabaco, foi proposto acompanhamento individual por meio de consultas com o médico e enfermeiro.

Tabela 3. Desfecho da condição tabágica de 40 participantes após tratamento na Atenção Primária à Saúde. Crateús, 2017

\begin{tabular}{lccc}
\hline Desfecho & Frequência $\left(f_{\mathrm{i}}\right)$ & Frequência relativa $\left(f_{\mathrm{ri}}\right)$ & Frequência percentual \\
\hline Abstinência & 27 & 0,675 & 67,5 \\
Recaída & 4 & 0,111 & 10 \\
Manutenção & 5 & 0,125 & 12,5 \\
Abandono & 4 & 0,1 & 10 \\
\hline Total & $\mathbf{4 0}$ & $\mathbf{1}$ & $\mathbf{1 0 0}$ \\
\hline
\end{tabular}

A implementação da abordagem e tratamento do tabagismo no âmbito da APS permitiu, num primeiro passo, realizar o diagnóstico situacional de uma população específica e em território determinado, processo importante para o levantamento de problemas e construção de ações qualificadas e abrangentes.

No contexto das redes de atenção à saúde, o enfrentamento da epidemia de tabagismo tem na APS seu lócus mais estratégico, dada a importância de seus quatro atributos principais: primeiro acesso, longitudinalidade, integralidade e coordenação do cuidado (BRASIL, 2015a).

Portanto, observou-se que, durante a identificação, avaliação e abordagem do usuário no território, a assistência à pessoa fumante no nível primário de atenção produziu resultados significativos, por entender de perto a realidade de vida das pessoas e sua relação estreita com o tabagismo, além de promover um cuidado compartilhado mediatizado por essa compreensão.

Do ponto de vista da caracterização sociodemográfica, o predomínio de mulheres, entre 
45 e 64 anos, e pessoas casadas, corrobora com outros estudos nacionais que avaliaram o perfil de fumantes que buscavam tratamento para cessação em serviço público (AZEVEDO, et al, 2009; BRASIL, 2015a; CASTRO; MATSUO; NUNES, 2010; SALES, et al, 2006; SATTLER, 2011; VIEIRA, 2014). Quanto à prevalência encontrada de pessoas com $1^{\circ}$ grau de instrução completo e incompleto, existe variação entre essa constatação e a literatura, apesar de ser concordante com a maioria dos estudos que encontraram taxas mais expressivas entre aqueles com menor grau de escolaridade (AZEVEDO, et al, 2009; BRASIL, 2015a; VIEIRA, 2014).

Sobre as variáveis associadas ao tabagismo, a maior prevalência (52,5\%) do nível de dependência à nicotina elevado (6-7 pontos) e muito elevado (8-10 pontos) é semelhante aos estudos de Sattler (2011) e Azevedo et al. (2009), que encontraram taxas de 52,5\% e 46,2\%, respectivamente.

Considerando os principais fatores motivacionais associados ao tabagismo, o predomínio da redução da tensão, dependência física e prazer de fumar na avaliação dos 40 participantes foram igualmente descritos no estudo de Vieira (2014), reputado como o pioneiro no Brasil a investigar, com base populacional, as motivações para o uso do tabaco.

A abordagem cognitivo-comportamental realizada em grupo permitiu acompanhar os usuários durante o período de 4 meses, tendo seguido a proposta de quatro sessões estruturadas, com periodicidade semanal, além de quatro encontros para seguimento de prevenção de recaída.

O grupo foi conduzido por quatro profissionais: o médico, exercendo a função de coordenador; e o psicólogo, fisioterapeuta e nutricionista, vinculados ao NASF, desempenhando o papel de observadores e facilitando a coordenação. Além disso, adequou-se o período de realização das sessões ao cronograma de atividades da unidade, de modo a permitir a participação do enfermeiro, técnico de enfermagem e agentes comunitários de saúde.

Destaca-se a relevância da atuação multiprofissional no tratamento do tabagismo, por entender que o cuidado à pessoa fumante envolve múltiplas intervenções, em que cada profissional se envolve a partir do nível de competência específico, contribuindo com a mudança comportamental a partir de uma atitude humanizada e da necessidade real de cada sujeito.

Durante os encontros, utilizou-se a abordagem ativa por meio da problematização, com ênfase nos principais conhecimentos que o fumante deve incorporar, que são: o significado da dependência física, psicológica e comportamental associadas ao tabagismo; o conceito de abstinência e tolerância; os benefícios de parar de fumar; a identificação e controle sobre as situações de gatilho que intensificam o desejo de fumar; a mudança de hábitos; o controle sobre 
os momentos de tensão e estresse (exercícios de respiração profunda e relaxamento muscular); o fortalecimento da decisão de abandonar o tabaco (técnicas de reforço) e de estar seguindo o melhor caminho (autoconfiança); e o significado de lapso e recaída, e como evitá-los (técnicas de enfrentamento).

Uma limitação do estudo deve ser considerada: o fato de não ter sido utilizado um período de tempo estendido após o fim do tratamento para a coleta de dados envolvendo o resultado terapêutico final. Assim, não foi possível determinar o percentual de abstinência definitiva com o tratamento implementado, que pode variar, entre os estudos, de cinco a doze meses, contados a partir da data de parada (BRASIL, 2015a).

Apesar da limitação, foi expressivamente alta a taxa de abstinência $(67,5 \%)$ ao final do grupo terapêutico, resultado superior a outros estudos (SALES, et al, 2006; SATTLER, 2011). Além disso, os baixos percentuais de manutenção, recaída e abandono se devem, provavelmente, à implementação de uma estratégia que envolveu múltiplas abordagens, planejamento compartilhado com o usuário e realização de acompanhamento intensivo.

\section{CONCLUSÃO}

Diante dos resultados apresentados, ressalta-se a importância da abordagem na APS com usuários tabagistas visto as contribuições que as intervenções trouxeram para o tratamento de controle do uso de substâncias. Encoraja-se assim, a realização de mais ações visando a diminuição do número de usuários.

As experiências comprovaram o impacto das ações de atenção à saúde no controle do tabagismo, com ênfase na APS em que se apresenta como espaço potente para abordagem de tal temática. Além disso, destaca-se a relevância dos profissionais da APS na abordagem, avaliação, motivação e acompanhamento adequados à pessoa tabagista, salientando também, o caráter multiprofissional apresentado pela equipe.

A intervenção, que se iniciou desde o reconhecimento da pessoa tabagista, teve várias etapas que permitiram com que houvesse uma abordagem efetiva. Dentre as etapas tiveram acolhimento e primeiro atendimento do usuário, estratificação quanto ao estágio motivacional para cessação tabágica, execução da estratégia de grupo para abandono do tabaco (dentre as quais foi utilizada a metodologia da problematização, proporcionando protagonismo dos participantes), e por fim, avaliação da necessidade e disponibilidade de terapia medicamentosa.

Portanto, espera-se que a intervenção, que proporcionou elevada taxa de abstinência ao tabaco, contribua com a sensibilização de gestores e trabalhadores da saúde para as grandes 
repercussões advindas do tabaco e a importância de seu controle, estimulando a criação de novos projetos e fortalecendo aqueles em execução.

\section{REFERÊNCIAS}

ACHARYA, A. et al. NCI Tobacco Control Monograph Series, 21 [Internet]. Bethesda (EstadosUnidos): U.S. Department of Health and Human Services, National Institutes of Health, National Cancer Institute; Genebra (Suíça): World Health Organization, 2016 Disponível em: http://cancercontrol.cancer.gov/brp/tcrb/monographs/21/docs/m21_complete.pdf. Acesso em: 10 ago. 2017.

AZEVEDO, R. C. S. et al. Grupo terapêutico para tabagistas: resultados após seguimento de dois anos. Revista da Associação Medica Brasileira, v. 55, n. 5, p. 593-596, 2009.

BRASIL. Ministério da Saúde (BR). Instituto Nacional de Câncer. Coordenação de Prevenção e Vigilância. Abordagem e tratamento do fumante: Consenso 2001. Rio de Janeiro: INCA; 2001.

BRASIL. Ministério da Saúde (BR). Secretaria de Atenção à Saúde. Departamento de Atenção Básica. Estratégias para o cuidado da pessoa com doença crônica: o cuidado da pessoa tabagista. Brasília: Ministério da Saúde; 2015a.

BRASIL. Ministério da Saúde (BR). Secretaria de Ciência, Tecnologia e Insumos Estratégicos. Departamento de Assistência Farmacêutica e Insumos Estratégicos. Relação Nacional de Medicamentos Essenciais: Rename 2014. 9. ed. Brasília: Ministério da Saúde; $2015 b$.

BRASIL. Ministério da Saúde. Portaria ${ }^{\circ}$ 1035, de 31 de maio de 2004. Amplia o acesso à abordagem e ao tratamento do tabagismo para a rede de atenção básica e de média complexidade do Sistema Único de Saúde.Diário Oficial da República Federativa do Brasil, Brasília (DF), 2004a jun1; Seção 1:24.

BRASIL. Ministério da Saúde. Portaria n 442, de 13 de agosto de 2004. Aprova o Plano para Implantação da Abordagem e Tratamento do Tabagismo no SUS e o Protocolo Clínico e Diretrizes Terapêuticas - Dependência à Nicotina.Diário Oficial da República Federativa do Brasil, Brasília (DF), 2004b ago17; Seção 1:62-5.

BRASIL. Ministério da Saúde. Portaria $\mathrm{n}^{\circ}$ 571, de 5 de abril de 2013. Atualiza as diretrizes de cuidado à pessoa tabagista no âmbito da Rede de Atenção à Saúde das Pessoas com Doenças Crônicas do Sistema Único de Saúde (SUS) e dá outras providências.Diário Oficial da República Federativa do Brasil, Brasília (DF), 2013 abr 8; Seção 1:56-7.

CAMPOS, P. C. M.; GOMIDE, M. O Programa Nacional de Controle do Tabagismo (PNCT) na perspectiva social: a análise de redes, capital e apoio social. Cadernos de Saúde Coletiva, v.23, n.4, p. 436-444, 2015. 
CARGNELUTTI, C. M.; BRUM, M. M.; ALÓS, A. P. A Alma cheia de bandeiras: ressignificação política e permanência de uma canção latino-americana. Anuário de Literatura, Florianópolis, v. 19, n. 2, p. 152-168, 2014.

CASTRO, M. R. P; MATSUO, T; NUNES, S. O. V. Características clínicas e qualidade de vida de fumantes em um centro de referência de abordagem e tratamento do tabagismo.

Jornal Brasileiro de Pneumologia, Brasília, v. 1, n. 36, p. 67-74, 2010.

FAGERSTRÖM, K. O. Measuring degree of physical dependence to tobacco smoking with reference to individualisation of treatment. Addictive Behaviors, v. 3, p. 235-241, 1978.

GIL, A. C. Como elaborar projetos de pesquisa. 5. ed. São Paulo: Atlas, 2010. 184p.

GIOVANELlA, L. Atenção Primária à Saúde seletiva ou abrangente?. Cad. Saúde Pública, Rio de Janeiro, v. 24, supl. 1, p. s21-s23, 2008.

PROCHASKA, J. O.; DICLEMENTE, C. C.; NORCROSS, J. C. In search of how people change: applications to addictive behaviors. American Psychology, Washington, DC, v. 47, n. 9, p. 1102, 1992.

REITSMA, M. B. et al. Smoking prevalence and attributable disease burden in 195 countries and territories, 1990-2015: a systematic analysis from the Global Burden of Disease Study 2015. Lancet, v. 388, p. 1659-1724, 2017.

ROMERO, L. C.; COSTA e SILVA, V. L. 23 Anos de Controle do Tabaco no Brasil: a Atualidade do Programa Nacional de Combate ao Fumo de 1988. Revista Brasileira de Cancerologia, Rio de Janeiro, v. 57, n. 3, p. 305-314, 2011.

SALES, M. P. U. et al. Ambulatório de apoio ao tabagista no Ceará: perfil dos pacientes e fatores associados ao sucesso terapêutico. Jornal Brasileiro de Pneumologia, v. 32, n.6, p. 410-417, 2006.

SATTLER, A. C. Prevalência da abstinência ao tabaco de pacientes tratados em unidades de saúde de Vitória-ES e fatores relacionados. 2011. 99 f. Dissertação (Mestrado em Saúde Coletiva) - Universidade Federal do Espírito Santo, Centro de Ciências da Saúde, Vitória, 2011.

SOUZA, E. S. T. de et al. Estrutura fatorial da versão brasileira da escala razões para fumar modificada. Revista da Associação Médica Brasileira, São Paulo, v. 55, n. 5, p. 557-562, 2009.

STARFIELD, B. Atenção Primária: equilíbrio entre necessidades de saúde, serviços e tecnologia. Brasília: UNESCO, Ministério da Saúde, 2002. 726 p.

VIEIRA, S.A.P. Prevalência de tabagismo e motivações para fumar: estudo de base populacional, em Florianópolis. 2014. Dissertação - Universidade Federal de Santa Catarina, Florianópolis, 2014. 